\title{
On the Inverting of A General Heptadiagonal Matrix
}

\author{
A. A. KARAWIA* \\ Computer science unit, Deanship of educational services, Qassim University, \\ P.O.Box 6595, Buraidah 51452, Saudi Arabia. \\ E-mail: kraoieh@qu.edu.sa
}

\begin{abstract}
In this paper, we developed new numeric and symbolic algorithms to find the inverse of any nonsingular heptadiagonal matrix. Symbolic algorithm will not break and it is without setting any restrictive conditions. The computational cost of our algorithms is $O(n)$. The algorithms are suitable for implementation using computer algebra system such as MAPLE, MATLAB and MATHEMATICA. Examples are given to illustrate the efficiency of the algorithms.
\end{abstract}

Keywords:Heptadiagonal matrices; LU factorization; Determinants; Computer algebra systems(CAS). AMS Subject Classification:15A15; 15A23; 68W30; 11Y05; 33F10; F.2.1; G.1.0.

\section{Introduction}

Then $\times n$ general heptadiagonal matrices take the form:

$$
H=\left(\begin{array}{cccccccccc}
d_{1} & e_{1} & f_{1} & g_{1} & & & & & & \\
c_{2} & d_{2} & e_{2} & f_{2} & g_{2} & & & & & \\
b_{3} & c_{3} & d_{3} & e_{3} & f_{3} & g_{3} & & 0 & & \\
a_{4} & b_{4} & c_{4} & d_{4} & e_{4} & f_{4} & g_{4} & & & \\
& & & & & & & & & \\
& & & & \ddots & \ddots & \ddots & \ddots & & \\
& & & a_{n-3} & b_{n-3} & c_{n-3} & d_{n-3} & e_{n-3} & f_{n-3} & g_{n-3} \\
& & & & a_{n-2} & b_{n-2} & c_{n-2} & d_{n-2} & e_{n-2} & f_{n-2} \\
& & & & & a_{n-1} & b_{n-1} & c_{n-1} & d_{n-1} & e_{n-1} \\
& & & & & & a_{n} & b_{n} & c_{n} & d_{n}
\end{array}\right), n>4 .
$$

where $\left\{a_{i}\right\}_{4 \leq i \leq n},\left\{b_{i}\right\}_{3 \leq i \leq n},\left\{c_{i}\right\}_{2 \leq i \leq n},\left\{d_{i}\right\}_{1 \leq i \leq n},\left\{e_{i}\right\}_{1 \leq i \leq n},\left\{f_{i}\right\}_{1 \leq i \leq n}$, and $\left\{g_{i}\right\}_{1 \leq i \leq n}$ are sequences of numbers such that $g_{i} \neq 0, \quad g_{n-2}=g_{n-1}=g_{n}=1$ and $f_{n-1}=f_{n}=e_{n}=0$.

Heptadiagonal matrices are frequently arise from boundary value problems. So a good technique for compute the inverse of such matrices is required. Also, These kind of matrices appear in many areas of science and engineering[1-9]. To the best of our knowledge, the inversion of a general heptadiagonal matrix of the form (1.1) has not been considered.

In [10], Karawia described a reliable symbolic computational algorithm for inverting general cyclic heptadiagonal matrices by using parallel computing along with recursion. An explicit formula for the determinant of a heptadiagonal symmetric matrix is given in[6]. Many researchers are studied special cases of heptadiagonal matrix. In [11], the authors presented a symbolic algorithm for finding the inverse of any general nonsingular tridiagonal matrix. A new efficient computational algorithm to find the inverse of a general tridiagonal matrix is presented in [12] based on the Doolittle LU factorization. In [13], the authors introduced a computationally efficient algorithm for obtaining the inverse of a tridiagonal matrix and a pentadiogonal matrix and they assumed a few conditions to avoid failure in their own algorithm. The

*Home Address: Mathematics Department, Faculty of Science, Mansoura University, Mansoura 35516, Egypt. Email:abibka@mans.edu.eg 
motivation of the current paper is to establish efficient algorithms for inverting heptadiagonal matrix. We generalized the algorithm[13] for finding the inverse of a general heptadiagonal matrix and we presented an efficient symbolic algorithm for finding the inverse of such matrices. The development of a symbolic algorithm is considered in order to remove all cases where the numeric algorithm fails.

The paper is organized as follows: In Section 2, Main result is presented. New numeric and symbolic algorithms are given in Section 3. In Section 4, Illustrative examples are presented. Conclusions of the work are given in Section 5.

\section{Main Result}

In this section, we present recurrence formulas for the columns of the inverse of a heptadiagonal matrix $H$. When the matrix $H$ is nonsingular, its inversion is computed as follows.

Let

$$
H^{-1}=\left[S_{i j}\right]_{1 \leq i, j \leq n}=\left[C_{1}, C_{2}, \ldots, C_{n}\right]
$$

where $C_{k}$ is the $k$ th column of the inverse matrix $H^{-1}$.

By using the fact $H H^{-1}=I_{n}$, where $I_{n}$ is the identity matrix, the first $(n-3)$ columns can be obtain by relations

$$
\left.\begin{array}{rl}
C_{n-3} & =\frac{1}{g_{n-3}}\left(E_{n}-d_{n} C_{n}-e_{n-1} C_{n-1}-f_{n-2} C_{n-2}\right), \\
C_{n-4} & =\frac{1}{g_{n-4}}\left(E_{n-1}-c_{n} C_{n}-d_{n-1} C_{n-1}-e_{n-2} C_{n-2}-f_{n-3} C_{n-3}\right), \\
C_{n-5} & =\frac{1}{g_{n-5}}\left(E_{n-2}-b_{n} C_{n}-c_{n-1} C_{n-1}-d_{n-2} C_{n-2}-e_{n-3} C_{n-3}-f_{n-4} C_{n-4}\right), \\
C_{j} & =\frac{1}{g_{j}}\left(E_{j+3}-a_{j+6} C_{j+6}-b_{j+5} C_{j+5}-c_{j+4} C_{j+4}-d_{j+3} C_{j+3}-e_{j+2} C_{j+2}-f_{j+1} C_{j+1}\right), \\
j & =n-6, n-7, \ldots, 1,
\end{array}\right\}
$$

where $E_{k}$ is the $k$ th unit vector.

From (2.1), we note that if we knowing the last three columns $C_{n}, C_{n-1}$, and $C_{n-2}$ then we can recursively compute the remaining $(n-3)$ columns $C_{n-3}, C_{n-4}, \ldots, C_{1}$.

At this point it is convenient to give recurrence formulas for computing $C_{n}, C_{n-1}$, and $C_{n-2}$.

Consider the sequence of numbers $\left\{A_{i}\right\}_{1 \leq i \leq n+3},\left\{B_{i}\right\}_{1 \leq i \leq n+3}$,and $\left\{C_{i}\right\}_{1 \leq i \leq n+3}$ characterized by a term recurrence relations

$$
\left.\begin{array}{l}
A_{1}=0, \\
A_{2}=0, \\
A_{3}=1, \\
d_{1} A_{1}+e_{1} A_{2}+f_{1} A_{3}+g_{1} A_{4}=0, \\
c_{2} A_{1}+d_{2} A_{2}+e_{2} A_{3}+f_{2} A_{4}+g_{2} A_{5}=0, \\
b_{3} A_{1}+c_{3} A_{2}+d_{3} A_{3}+e_{3} A_{4}+f_{3} A_{5}+g_{3} A_{6}=0, \\
a_{i} A_{i-3}+b_{i} A_{i-2}+c_{i} A_{i-1}+d_{i} A_{i}+e_{i} A_{i+1}+f_{i} A_{i+2}+g_{i} A_{i+3}=0, \quad i \geq 4,
\end{array}\right\}
$$




$$
\left.\begin{array}{l}
B_{1}=0, \\
B_{2}=1, \\
B_{3}=0 \\
d_{1} B_{1}+e_{1} B_{2}+f_{1} B_{3}+g_{1} B_{4}=0, \\
c_{2} B_{1}+d_{2} B_{2}+e_{2} B_{3}+f_{2} B_{4}+g_{2} B_{5}=0, \\
b_{3} B_{1}+c_{3} B_{2}+d_{3} B_{3}+e_{3} B_{4}+f_{3} B_{5}+g_{3} B_{6}=0, \\
a_{i} B_{i-3}+b_{i} B_{i-2}+c_{i} B_{i-1}+d_{i} B_{i}+e_{i} B_{i+1}+f_{i} B_{i+2}+g_{i} B_{i+3}=0, \quad i \geq 4,
\end{array}\right\}
$$

and

$$
\left.\begin{array}{l}
C_{1}=1, \\
C_{2}=0, \\
C_{3}=0, \\
d_{1} C_{1}+e_{1} C_{2}+f_{1} C_{3}+g_{1} C_{4}=0, \\
c_{2} C_{1}+d_{2} C_{2}+e_{2} C_{3}+f_{2} C_{4}+g_{2} C_{5}=0, \\
b_{3} C_{1}+c_{3} C_{2}+d_{3} C_{3}+e_{3} C_{4}+f_{3} C_{5}+g_{3} C_{6}=0, \\
a_{i} C_{i-3}+b_{i} C_{i-2}+c_{i} C_{i-1}+d_{i} C_{i}+e_{i} C_{i+1}+f_{i} C_{i+2}+g_{i} C_{i+3}=0, \quad i \geq 4 .
\end{array}\right\}
$$

Now, we can give matrix forms for term recurrences (2.2), (2.3) and (2.4)

$$
\begin{aligned}
& \mathbf{H A}=-A_{n+1} E_{n-2}-A_{n+2} E_{n-1}-A_{n+3} E_{n}, \\
& \mathbf{H B}=-B_{n+1} E_{n-2}-B_{n+2} E_{n-1}-B_{n+3} E_{n}, \\
& \mathbf{H C}=-C_{n+1} E_{n-2}-C_{n+2} E_{n-1}-C_{n+3} E_{n},
\end{aligned}
$$

where $\mathbf{A}=\left[A_{1}, A_{2}, \ldots, A_{n}\right]^{t}, \mathbf{B}=\left[B_{1}, B_{2}, \ldots, B_{n}\right]^{t}$, and $\mathbf{C}=\left[C_{1}, C_{2}, \ldots, C_{n}\right]^{t}$.

Let's define the following determinants:

$$
\begin{aligned}
X_{i} & =\left|\begin{array}{lll}
A_{i} & A_{n+2} & A_{n+3} \\
B_{i} & B_{n+2} & B_{n+3} \\
C_{i} & C_{n+2} & C_{n+3}
\end{array}\right|, \quad i=1,2, \ldots, n+1, \\
Y_{i} & =\left|\begin{array}{lll}
A_{i} & A_{n+1} & A_{n+3} \\
B_{i} & B_{n+1} & B_{n+3} \\
C_{i} & C_{n+1} & C_{n+3}
\end{array}\right|, \quad i=1,2, \ldots, n+2, \\
Z_{i} & =\left|\begin{array}{lll}
A_{i} & A_{n+1} & A_{n+2} \\
B_{i} & B_{n+1} & B_{n+2} \\
C_{i} & C_{n+1} & C_{n+2}
\end{array}\right|, \quad i=1,2, \ldots, n+3 .
\end{aligned}
$$

By simple calculations, we have

$$
\begin{gathered}
\mathbf{H X}=-X_{n+1} E_{n-2}, \\
\mathbf{H Y}=-Y_{n+2} E_{n-1}, \\
\mathbf{H Z}=-Z_{n+3} E_{n},
\end{gathered}
$$

where $\mathbf{X}=\left[X_{1}, X_{2}, \ldots, X_{n}\right]^{t}, \mathbf{Y}=\left[Y_{1}, Y_{2}, \ldots, Y_{n}\right]^{t}$, and $\mathbf{Z}=\left[Z_{1}, Z_{2}, \ldots, Z_{n}\right]^{t}$.

Remark 2.1. $X_{n+1}=-Y_{n+2}=Z_{n+3}$.

Lemma 2.1. (generalization version of Lemma 3.1 in [13]) If $X_{n+1}=0$, then the matrix $\mathbf{H}$ is singular.

Proof. The proof is simple. 
Theorem 2.1. (generalization version of theorem 3.1 in [13]) Suppose that $X_{n+1} \neq 0$, then $\mathbf{H}$ is invertible and

$$
\begin{gathered}
C_{n}=\left[\frac{-Z_{1}}{Z_{n+3}}, \frac{-Z_{2}}{Z_{n+3}}, \ldots, \frac{-Z_{n}}{Z_{n+3}}\right]^{t}, \\
C_{n-1}=\left[\frac{-Y_{1}}{Y_{n+2}}, \frac{-Y_{2}}{Y_{n+2}}, \ldots, \frac{-Y_{n}}{Y_{n+2}}\right]^{t}, \\
C_{n-2}=\left[\frac{-X_{1}}{X_{n+1}}, \frac{-X_{2}}{X_{n+1}}, \ldots, \frac{-X_{n}}{X_{n+1}}\right]^{t} .
\end{gathered}
$$

Proof. Since $\operatorname{det}(\mathbf{H})=-\left(\prod_{i=1}^{n-3} g_{i}\right) X_{n+1} \neq 0$, then $\mathbf{H}$ is invertible. From (2.11), (2.12) and (2.13) we obtain $C_{n}, C_{n-1}$, and $C_{n-2}$. The proof is completed.

\section{New numeric and symbolic algorithms for the inverse of hep- tadiagonal matrix}

In this section, we formulate the result in the previous section. It is a numerical algorithm to compute the inverse of a general heptadiagonal matrix of the form (1.1) when it exists.

Algorithm 3.1 To find the inverse of heptadiagonal matrix (1.1).

let $f_{n-1}=f_{n}=e_{n}=0$ and $g_{n-2}=g_{n-1}=g_{n}$.

INPUT: Order of the matrix $n$ and the components $a_{i}, b_{j}, c_{k}, d_{l}, e_{l}, f_{l}$, and $g_{l}$ for $i=4,5, \ldots, n$, $j=3,4, \ldots, n, k=2,3, \ldots, n$, and $l=1,2, \ldots, n$,

OUTPUT: The inverse of heptadiagonal matrix $\mathbf{H}^{-1}$.

Step 1: Compute the sequence of numbers $A_{i}, B_{i}$, and $C_{i}$ for $i=1,2, \ldots, n+3$ using (2.2), (2.3) and (2.4) respectively.

Step 2: Compute $X_{i}, \quad i=1,2, \ldots, n+1$ using (2.8), $Y_{i}, \quad i=1,2, \ldots, n+2$ using (2.9) and $Z_{i}$, $i=1,2, \ldots, n+3$ using (2.10).

Step 3: Compute the last three columns $C_{n}, C_{n-1}$, and $C_{n-2}$ using (2.14), (2.15), and (2.16) respectively.

Step 4: Compute the remaining (n-3)-columns $C_{j}, \quad j=n-3, n-4, \ldots, 1$ using (2.1).

Step 5: Set $\mathbf{H}^{-1}=\left[C_{1}, C_{2}, \ldots, C_{n}\right]$.

The numeric algorithm 3.1 will be referred to as NINVHEPTA algorithm. The computational cost of NINVHEPTA algorithm is $103 n+69$ operations.

As can be easily seen, it breaks down unless the conditions $g_{i} \neq 0$ are satisfied for all $i=1,2, \ldots, n-3$. So the following symbolic algorithm is developed in order to remove the cases where the numeric algorithm fails.

Algorithm 3.2 To find the inverse of heptadiagonal matrix (1.1).

let $f_{n-1}=f_{n}=e_{n}=0$ and $g_{n-2}=g_{n-1}=g_{n}$.

INPUT: Order of the matrix $n$ and the components $a_{i}, b_{j}, c_{k}, d_{l}, e_{l}, f_{l}$, and $g_{l}$ for $i=4,5, \ldots, n$, $j=3,4, \ldots, n, k=2,3, \ldots, n$, and $l=1,2, \ldots, n$,

OUTPUT: The inverse of heptadiagonal matrix $\mathbf{H}^{-1}$.

Step 1: If $g_{i}=0$ for any $i=1,2, \ldots, n-3$ set $g_{i}=t(t$ is just a symbolic name).

Step 2: Compute the sequence of numbers $A_{i}, B_{i}$, and $C_{i}$ for $i=1,2, \ldots, n+3$ using (2.2), (2.3) and (2.4) respectively. 
Step 3: Compute $X_{i}, \quad i=1,2, \ldots, n+1$ using (2.8), $Y_{i}, \quad i=1,2, \ldots, n+2$ using (2.9) and $Z_{i}$, $i=1,2, \ldots, n+3$ using (2.10).

Step 4: Compute the last three columns $C_{n}, C_{n-1}$, and $C_{n-2}$ using (2.14), (2.15), and (2.16) respectively.

Step 5: Compute the remaining (n-3)-columns $C_{j}, \quad j=n-3, n-4, \ldots, 1$ using (2.1).

Step 6: Substitute the actual value $t=0$ in all expressions to obtain the elements of columns $C_{j}$, $j=1,2, \ldots, n$.

Step 7: Set $\mathbf{H}^{-1}=\left[C_{1}, C_{2}, \ldots, C_{n}\right]$.

The symbolic algorithm 3.2 will be referred to as SINVHEPTA algorithm. The computational cost of SINVHEPTA algorithm is $103 n+69$ operations. Based on SINVHEPTA algorithm, a MAPLE procedure for inverting a general nonsingular heptadiagonal matrix $\mathbf{H}$ is listed as an Appendix.

\section{ILLUSTRATIVE EXAMPLES}

In this section we give three examples for the sake of illustration.

Example 4.1. (Case I: $g_{i} \neq 0$ for all $i$ )

Find the inverse of following $10 \times 10$ heptadiagonal matrix

$$
H_{1}=\left[\begin{array}{cccccccccc}
2 & 1 & 4 & -1 & 0 & 0 & 0 & 0 & 0 & 0 \\
5 & 1 & 1 & 2 & 2 & 0 & 0 & 0 & 0 & 0 \\
1 & 2 & -3 & 2 & 7 & 2 & 0 & 0 & 0 & 0 \\
6 & 1 & 3 & 2 & 3 & -1 & 3 & 0 & 0 & 0 \\
0 & 1 & -1 & 2 & 2 & -3 & 4 & 1 & 0 & 0 \\
0 & 0 & 4 & 4 & 4 & 1 & 2 & 1 & 1 & 0 \\
0 & 0 & 0 & -1 & 2 & -1 & 3 & -3 & 2 & 1 \\
0 & 0 & 0 & 0 & 3 & 1 & 2 & 1 & 11 & 3 \\
0 & 0 & 0 & 0 & 0 & 4 & -3 & 2 & 1 & 1 \\
0 & 0 & 0 & 0 & 0 & 0 & -7 & 1 & 1 & 2
\end{array}\right]
$$

Solution: By applying the NINVHEPTA algorithm, it yields

- Step 1: $A=\left[0,0,1,4,-\frac{9}{2}, \frac{53}{4}, \frac{21}{4}, \frac{83}{4},-\frac{93}{2}, \frac{663}{4},-\frac{67}{4},-198,-269\right]$,

$$
\begin{aligned}
& B=\left[0,1,0,1,-\frac{3}{2}, \frac{13}{4}, \frac{19}{12}, \frac{41}{12},-\frac{47}{6}, \frac{341}{12},-\frac{53}{12},-\frac{107}{3},-\frac{124}{3}\right], \text { and } \\
& C=\left[1,0,0,2,-\frac{9}{2}, \frac{53}{4}, \frac{67}{12}, \frac{269}{12},-\frac{221}{6}, \frac{1781}{12},-\frac{881}{12},-\frac{578}{3},-\frac{730}{3}\right] .
\end{aligned}
$$

- Step 2: $X=\left[\frac{4231}{3},-\frac{10942}{3},-\frac{2146}{3},-3688, \frac{4687}{2},-\frac{31741}{12},-\frac{19873}{12}, \frac{51721}{12}, \frac{19773}{2},-\frac{154735}{12},-\frac{905413}{12}\right]$,

$$
\begin{gathered}
Y=\left[\frac{1983}{4},-\frac{62693}{4}, \frac{11759}{6},-\frac{82109}{12}, \frac{49839}{4},-\frac{220819}{12},-\frac{141107}{12},-\frac{5312}{3}, \frac{160577}{12},-\frac{563539}{12}, 0, \frac{905413}{12}\right], \text { and } \\
Z=\left[\frac{3325}{12},-\frac{33928}{3}, \frac{21211}{12},-\frac{22109}{6}, 7763,-\frac{19327}{2},-\frac{84955}{12}, \frac{50981}{12},-\frac{15235}{4}, \frac{76363}{6}, 0,0,-\frac{905413}{12}\right] .
\end{gathered}
$$

- Step 3: $C_{10}=\left[\frac{3325}{905413},-\frac{135712}{905413}, \frac{21211}{905413},-\frac{44218}{905413}, \frac{93156}{905413},-\frac{115962}{905413},-\frac{84955}{905413}, \frac{50981}{905413},-\frac{45705}{905413}, \frac{152726}{905413}\right]^{t}$,

$$
\begin{aligned}
& C_{9}=\left[-\frac{5949}{905413}, \frac{188079}{905413},-\frac{23518}{905413}, \frac{82109}{905413},-\frac{149517}{905413}, \frac{220819}{905413}, \frac{141107}{905413}, \frac{21248}{905413},-\frac{160577}{905413}, \frac{563539}{905413}\right]^{t} \text {, and } \\
& C_{8}=\left[\frac{16924}{905413},-\frac{43768}{905413},-\frac{8584}{905413},-\frac{44256}{905413}, \frac{28122}{905413},-\frac{31741}{905413},-\frac{19873}{905413}, \frac{51721}{905413}, \frac{118638}{905413},-\frac{154735}{905413}\right]^{t} .
\end{aligned}
$$

- Step 4: $C_{7}=\left[-\frac{51473}{905413}, \frac{214649}{905413}, \frac{6848}{905413}, \frac{139095}{905413},-\frac{121161}{905413}, \frac{106328}{905413}, \frac{88422}{905413},-\frac{278373}{905413},-\frac{103927}{905413}, \frac{500627}{905413},\right]^{t}$, 


$$
\begin{aligned}
& C_{6}=\left[-\frac{80594}{905413},-\frac{217}{905413}, \frac{83035}{905413}, \frac{170735}{905413},-\frac{10659}{905413}, \frac{31638}{905413},-\frac{14393}{905413},-\frac{84414}{905413}, \frac{14531}{905413},-\frac{15434}{905413}\right]^{t}, \\
& C_{5}=\left[-\frac{82176}{905413}, \frac{447486}{905413},-\frac{28082}{905413}, \frac{170806}{905413},-\frac{175068}{905413},-\frac{6589}{905413}, \frac{102273}{905413}, \frac{9510}{905413},-\frac{78091}{905413}, \frac{392246}{905413}\right]^{t}, \\
& C_{4}=\left[\frac{205297}{905413},-\frac{910556}{905413}, \frac{6935}{905413},-\frac{472222}{905413}, \frac{410790}{905413},-\frac{147233}{905413}, \frac{42741}{905413}, \frac{427692}{905413},-\frac{147953}{905413}, \frac{9724}{905413}\right]^{t}, \\
& C_{3}=\left[-\frac{2619}{905413},-\frac{30890}{905413},-\frac{25421}{905413},-\frac{137812}{905413}, \frac{172515}{905413},-\frac{19216}{905413},-\frac{46090}{905413}, \frac{62775}{905413}, \frac{11493}{905413},-\frac{198449}{905413}\right]^{t}, \\
& C_{2}=\left[-\frac{29328}{905413}, \frac{877900}{905413},-\frac{53389}{905413}, \frac{605688}{905413},-\frac{491917}{905413}, \frac{172702}{905413},-\frac{34896}{905413},-\frac{501141}{905413}, \frac{156703}{905413}, \frac{50083}{905413},\right]^{t}, \text { and } \\
& C_{1}=\left[-\frac{88555}{905413}, \frac{552363}{905413}, \frac{125378}{905413},-\frac{28648}{905413},-\frac{88835}{905413}, \frac{19552}{905413},-\frac{17938}{905413},-\frac{61611}{905413}, \frac{46355}{905413},-\frac{55155}{905413}\right]^{t} .
\end{aligned}
$$

- Step 5: $H_{1}^{-1}=$

$\left[\begin{array}{cccccccccc}-\frac{88555}{905413} & -\frac{29328}{905413} & -\frac{2619}{905413} & \frac{205297}{905413} & -\frac{82176}{905413} & -\frac{80594}{905413} & -\frac{51473}{905413} & \frac{16924}{905413} & -\frac{5949}{905413} & \frac{3325}{905413} \\ \frac{552363}{905413} & \frac{877900}{905413} & -\frac{30890}{905413} & -\frac{910556}{905413} & \frac{447486}{905413} & -\frac{217}{905413} & \frac{214649}{905413} & -\frac{43768}{905413} & \frac{188079}{905413} & -\frac{135712}{905413} \\ \frac{125378}{905413} & -\frac{53389}{905413} & -\frac{25421}{905413} & \frac{6935}{905413} & -\frac{28082}{905413} & \frac{83035}{905413} & \frac{6848}{905413} & -\frac{8584}{905413} & -\frac{23518}{905413} & \frac{21211}{905413} \\ -\frac{28648}{905413} & \frac{605688}{905413} & -\frac{137812}{905413} & -\frac{472222}{905413} & \frac{170806}{905413} & \frac{170735}{905413} & \frac{139095}{905413} & -\frac{44256}{905413} & \frac{82109}{905413} & -\frac{44218}{905413} \\ -\frac{88835}{905413} & -\frac{491917}{905413} & \frac{172515}{905413} & \frac{410790}{905413} & -\frac{175068}{905413} & -\frac{10659}{905413} & -\frac{121161}{905413} & \frac{28122}{905413} & -\frac{149517}{905413} & \frac{93156}{905413} \\ \frac{19552}{905413} & \frac{172702}{905413} & -\frac{19216}{905413} & -\frac{147233}{905413} & -\frac{6589}{905413} & \frac{31638}{905413} & \frac{106328}{905413} & -\frac{31741}{905413} & \frac{220819}{905413} & -\frac{115962}{905413} \\ -\frac{17938}{905413} & -\frac{34896}{905413} & -\frac{46090}{905413} & \frac{42741}{905413} & \frac{102273}{905413} & -\frac{14393}{905413} & \frac{88422}{905413} & -\frac{19873}{905413} & \frac{141107}{905413} & -\frac{84955}{905413} \\ -\frac{61611}{905413} & -\frac{501141}{905413} & \frac{62775}{905413} & \frac{427692}{905413} & \frac{9510}{905413} & -\frac{84414}{905413} & -\frac{278373}{905413} & \frac{51721}{905413} & \frac{21248}{905413} & \frac{50981}{905413} \\ \frac{46355}{905413} & \frac{156703}{905413} & \frac{11493}{905413} & -\frac{147953}{905413} & -\frac{78091}{905413} & \frac{14531}{905413} & -\frac{103927}{905413} & \frac{118638}{905413} & -\frac{160577}{905413} & -\frac{45705}{905413} \\ -\frac{55155}{905413} & \frac{50083}{905413} & -\frac{198449}{905413} & \frac{9724}{905413} & \frac{392246}{905413} & -\frac{15434}{905413} & \frac{500627}{905413} & -\frac{154735}{905413} & \frac{563539}{905413} & \frac{152726}{905413}\end{array}\right]$

Example 4.2.(Case II: $g_{i}=0$ for at least one of $i$ )

Find the inverse of following $5 \times 5$ heptadiagonal matrix

$$
H_{2}=\left[\begin{array}{ccccc}
2 & 3 & 4 & 1 & 0 \\
-1 & 1 & -2 & 3 & 0 \\
3 & 5 & 1 & -1 & 2 \\
4 & -1 & 3 & 2 & 6 \\
0 & 2 & 1 & 4 & -3
\end{array}\right]
$$

\section{Solution:}

i- By applying the NINVHEPTA algorithm, it breaks down since $g_{2}=0$.

ii- By applying the SINVHEPTA algorithm, it yields

- Step 1: $A=\left[0,0,1,-4,14 x^{-1},-\frac{5 x+28}{x}, \frac{5 x-84}{x}, 3 \frac{5 x+14}{x}\right]$,

$$
\begin{aligned}
& B=\left[0,1,0,-3,8 x^{-1},-8 \frac{x+2}{x}, \frac{7 x-48}{x}, 2 \frac{5 x+12}{x}\right], \text { and } \\
& C=\left[1,0,0,-2,7 x^{-1},-\frac{5 x+14}{x},-42 x^{-1}, \frac{8 x+21}{x}\right] .
\end{aligned}
$$

- Step 2: $X=\left[\frac{55 x+294}{x}, \frac{63+40 x}{x},-\frac{183+56 x}{x},-3 \frac{2 x+15}{x},-79 x^{-1},-\frac{315 x+901}{x}\right]$,

$$
\begin{aligned}
& Y=\left[-2 \frac{35 x+88}{x}, 7 \frac{5 x+13}{x}, 2 \frac{7 x+18}{x},-\frac{21 x+65}{x},-14 x^{-1}, 0, \frac{315 x+901}{x}\right], \text { and } \\
& Z=\left[-\frac{5 x-548}{x}, 5 \frac{5 x-28}{x},-\frac{35 x+194}{x}, 25 \frac{3 x+4}{x},-325 x^{-1}, 0,0,-\frac{315 x+901}{x}\right] .
\end{aligned}
$$

- Step 3: $C_{5}=\left[-\frac{5 x-548}{315 x+901}, 5 \frac{5 x-28}{315 x+901},-\frac{35 x+194}{315 x+901}, 25 \frac{3 x+4}{315 x+901},-325 \frac{315}{x+901}\right]^{t}$,

$$
\begin{aligned}
& C_{4}=\left[2 \frac{35 x+88}{315 x+901},-7 \frac{5 x+13}{315 x+901},-2 \frac{7 x+18}{315 x+901}, \frac{21 x+65}{315 x+901}, 14 \frac{315}{x+901}\right]^{t}, \text { and } \\
& C_{3}=\left[\frac{55 x+294}{315 x+901}, \frac{63+40 x}{315 x+901},-\frac{183+56 x}{315 x+901},-3 \frac{2 x+15}{315 x+901}-79 \frac{315}{x+901}\right]^{t} .
\end{aligned}
$$


- Step 4: $C_{2}=\left[-545 \frac{315}{x+901}, 205 \frac{315}{x+901}, 91 \frac{315}{x+901}, 111 \frac{315}{x+901}, 315 \frac{315}{x+901}\right]^{t}$, and

$$
C_{1}=\left[-5 \frac{13 x+123}{315 x+901}, 10 \frac{x+19}{315 x+901}, 56 \frac{2 x+7}{315 x+901},-\frac{33 x+7}{315 x+901}, 248 \frac{315}{x+901}\right]^{t} .
$$

- Step 5: $H_{2}^{-1}=$

$$
\left[\begin{array}{ccccc}
-5 \frac{13 x+123}{315 x+901} & -545 \frac{315}{x+901} & \frac{55 x+294}{315 x+901} & 2 \frac{35 x+88}{315 x+901} & -\frac{5 x-548}{315 x+901} \\
10 \frac{x+19}{315 x+901} & 205 \frac{315}{x+901} & \frac{63+40 x}{315 x+901} & -7 \frac{5 x+13}{315 x+901} & 5 \frac{5 x-28}{315 x+901} \\
56 \frac{2 x+7}{315 x+901} & 91 \frac{315}{x+901} & -\frac{183+56 x}{315 x+901} & -2 \frac{7 x+18}{315 x+901} & -\frac{35 x+194}{315 x+901} \\
-\frac{33 x+7}{315 x+901} & 111 \frac{315}{x+901} & -3 \frac{2 x+15}{315 x+901} & \frac{21 x+65}{315 x+901} & 25 \frac{3 x+4}{315 x+901} \\
248 \frac{315}{x+901} & 315 \frac{315}{x+901} & -79 \frac{315}{x+901} & 14 \frac{315}{x+901} & -325 \frac{315}{x+901}
\end{array}\right]_{x=0}
$$

- Step 6: $H_{2}^{-1}=\left[\begin{array}{ccccc}-\frac{615}{901} & -\frac{545}{901} & \frac{294}{901} & \frac{176}{901} & \frac{548}{901} \\ \frac{190}{901} & \frac{205}{901} & \frac{63}{901} & -\frac{91}{901} & -\frac{140}{901} \\ \frac{392}{901} & \frac{91}{901} & -\frac{183}{901} & -\frac{36}{901} & -\frac{194}{901} \\ -\frac{7}{901} & \frac{111}{901} & -\frac{45}{901} & \frac{65}{901} & \frac{100}{901} \\ \frac{248}{901} & \frac{315}{901} & -\frac{79}{901} & \frac{14}{901} & -\frac{325}{901}\end{array}\right]$.

Example 4.3. We consider the following $n \times n$ heptadiagonal matrix in order to demonstrate the efficiency of SINVHEPTA algorithm.

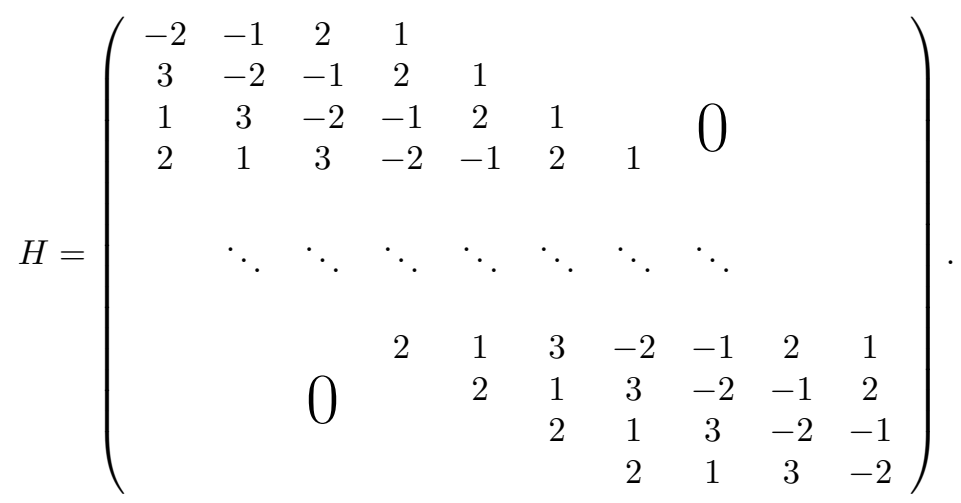

In Table 1. we give a comparison of the running time between SINVHEPTA algorithm and MatrixInverse function in Maple 13.0 for different orders. It was tested in an Intel(R) Core(TM) i7-4700MQ CPU@2.40GHz 2.40 GHz.

Table1.

Running time(in Seconds) of proposed algorithm and MatrixInverse function in Maple 13.0.

\begin{tabular}{|c|c|c|}
\hline $\mathrm{n}$ & SINVHEPTA algorithm & MatrixInverse function in Maple 13.0 \\
\hline 100 & 1.282 & 1.672 \\
\hline 200 & 3.078 & 11.687 \\
\hline 300 & 9.719 & 40.625 \\
\hline 500 & 44.312 & 208.968 \\
\hline 1000 & 439.578 & 2441.188 \\
\hline
\end{tabular}

\section{CONCLUSIONS}

In this work new numeric and symbolic algorithms have been developed for finding the inverse of any nonsingular heptadiagonal matrix. The algorithms are reliable, computationally efficient and the symbolic algorithm removes the cases where the numeric algorithms fail. 
$>$ restart:with(LinearAlgebra):

hepta_inv := proc(n::posint,a::vector,b::vector,c::vector,d::vector,e::vector,f::vector,g::vector)

local i,j;

global A,B,C,X,Y,Z,S,Hinv;

$\mathrm{A}:=\operatorname{array}(1 \ldots \mathrm{n}+3): \mathrm{B}:=\operatorname{array}(1 \ldots \mathrm{n}+3): \mathrm{C}:=\operatorname{array}(1 \ldots \mathrm{n}+3): \mathrm{X}:=\operatorname{array}(1 \ldots \mathrm{n}+1)$ :

$\mathrm{Y}:=\operatorname{array}(1 \ldots \mathrm{n}+2): \mathrm{Z}:=\operatorname{array}(1 \ldots \mathrm{n}+3): \mathrm{S}:=\operatorname{array}(1 . . \mathrm{n}, 1 . . \mathrm{n}, \mathrm{sparse})$ :

for i from 1 to n-3 do

end do:

$$
\text { if } \mathrm{g}[\mathrm{i}]=0 \text { then } \mathrm{g}[\mathrm{i}]:=\mathrm{x} \text { end if }
$$

$\mathrm{A}[1]:=0: \mathrm{A}[2]:=0: \mathrm{A}[3]:=1: \mathrm{B}[1]:=0: \mathrm{B}[2]:=1: \mathrm{B}[3]:=0: \mathrm{C}[1]:=1: \mathrm{C}[2]:=0: \mathrm{C}[3]:=0:$

$\mathrm{A}[4]:=-\operatorname{simplify}(\mathrm{f}[1] / \mathrm{g}[1]): \mathrm{A}[5]:=-\operatorname{simplify}((\mathrm{e}[2]+\mathrm{A}[4] * \mathrm{f}[2]) / \mathrm{g}[2]): \mathrm{A}[6]:=-\operatorname{simplify}((\mathrm{d}[3]+\mathrm{e}[3] * \mathrm{~A}[4]+\mathrm{A}[5] * \mathrm{f}[3]) / \mathrm{g}[3])$ :

$\mathrm{B}[4]:=-\operatorname{simplify}(\mathrm{e}[1] / \mathrm{g}[1]): \mathrm{B}[5]:=-\operatorname{simplify}\left(\left(\mathrm{d}[2]+\mathrm{B}[4]^{*} \mathrm{f}[2]\right) / \mathrm{g}[2]\right): \mathrm{B}[6]:=-\operatorname{simplify}((\mathrm{c}[3]+\mathrm{e}[3] * \mathrm{~B}[4]+\mathrm{B}[5] * \mathrm{f}[3]) / \mathrm{g}[3])$ :

$\mathrm{C}[4]:=-\operatorname{simplify}(\mathrm{d}[1] / \mathrm{g}[1]): \mathrm{C}[5]:=-\operatorname{simplify}\left(\left(\mathrm{c}[2]+\mathrm{C}[4]^{*} \mathrm{f}[2]\right) / \mathrm{g}[2]\right): \mathrm{C}[6]:=-\operatorname{simplify}\left(\left(\mathrm{b}[3]+\mathrm{e}[3] * \mathrm{C}[4]+\mathrm{C}[5]^{*} \mathrm{f}[3]\right) / \mathrm{g}[3]\right)$ :

for i from 4 to $\mathrm{n}$ do

$$
\begin{aligned}
& \mathrm{A}[\mathrm{i}+3]:=-\operatorname{simplify}\left(\left(\mathrm{a}[\mathrm{i}]^{*} \mathrm{~A}[\mathrm{i}-3]+\mathrm{b}[\mathrm{i}]^{*} \mathrm{~A}[\mathrm{i}-2]+\mathrm{c}[\mathrm{i}]^{*} \mathrm{~A}[\mathrm{i}-1]+\mathrm{d}[\mathrm{i}]^{*} \mathrm{~A}[\mathrm{i}]+\mathrm{e}[\mathrm{i}]^{*} \mathrm{~A}[\mathrm{i}+1]+\mathrm{f}[\mathrm{i}]^{*} \mathrm{~A}[\mathrm{i}+2]\right) / \mathrm{g}[\mathrm{i}]\right): \\
& \mathrm{B}[\mathrm{i}+3]:=-\operatorname{simplify}\left(\left(\mathrm{a}[\mathrm{i}]^{*} \mathrm{~B}[\mathrm{i}-3]+\mathrm{b}[\mathrm{i}]^{*} \mathrm{~B}[\mathrm{i}-2]+\mathrm{c}\left[\mathrm{i}{ }^{*} \mathrm{~B}[\mathrm{i}-1]+\mathrm{d}[\mathrm{i}]^{*} \mathrm{~B}[\mathrm{i}]+\mathrm{e}[\mathrm{i}]^{*} \mathrm{~B}[\mathrm{i}+1]+\mathrm{f}[\mathrm{i}]^{*} \mathrm{~B}[\mathrm{i}+2]\right) / \mathrm{g}[\mathrm{i}]\right):\right. \\
& \mathrm{C}[\mathrm{i}+3]:=-\operatorname{simplify}\left(\left(\mathrm{a}[\mathrm{i}]^{*} \mathrm{C}[\mathrm{i}-3]+\mathrm{b}[\mathrm{i}]^{*} \mathrm{C}[\mathrm{i}-2]+\mathrm{c}[\mathrm{i}]^{*} \mathrm{C}[\mathrm{i}-1]+\mathrm{d}[\mathrm{i}]^{*} \mathrm{C}[\mathrm{i}]+\mathrm{e}[\mathrm{i}]^{*} \mathrm{C}[\mathrm{i}+1]+\mathrm{f}[\mathrm{i}]^{*} \mathrm{C}[\mathrm{i}+2]\right) / \mathrm{g}[\mathrm{i}]\right):
\end{aligned}
$$

end do:

$\mathrm{i}:=$ 'i':

for $\mathrm{i}$ from 1 to $\mathrm{n}+1$ do

$\mathrm{X}[\mathrm{i}]:=\operatorname{simplify}(\operatorname{Determinant}(\operatorname{Matrix}([[\mathrm{A}[\mathrm{n}+3], \mathrm{A}[\mathrm{n}+2], \mathrm{A}[\mathrm{i}]],[\mathrm{B}[\mathrm{n}+3], \mathrm{B}[\mathrm{n}+2], \mathrm{B}[\mathrm{i}]],[\mathrm{C}[\mathrm{n}+3], \mathrm{C}[\mathrm{n}+2], \mathrm{C}[\mathrm{i}]]])))$ :

$\mathrm{Y}[\mathrm{i}]:=\operatorname{simplify}(\operatorname{Determinant}(\operatorname{Matrix}([[\mathrm{A}[\mathrm{n}+3], \mathrm{A}[\mathrm{n}+1], \mathrm{A}[\mathrm{i}]],[\mathrm{B}[\mathrm{n}+3], \mathrm{B}[\mathrm{n}+1], \mathrm{B}[\mathrm{i}]],[\mathrm{C}[\mathrm{n}+3], \mathrm{C}[\mathrm{n}+1], \mathrm{C}[\mathrm{i}]]])))$ :

$\mathrm{Z}[\mathrm{i}]:=\operatorname{simplify}(\operatorname{Determinant}(\operatorname{Matrix}([[\mathrm{A}[\mathrm{n}+2], \mathrm{A}[\mathrm{n}+1], \mathrm{A}[\mathrm{i}]],[\mathrm{B}[\mathrm{n}+2], \mathrm{B}[\mathrm{n}+1], \mathrm{B}[\mathrm{i}]],[\mathrm{C}[\mathrm{n}+2], \mathrm{C}[\mathrm{n}+1], \mathrm{C}[\mathrm{i}]]])))$ : end do:

$\mathrm{Y}[\mathrm{n}+2]:=\operatorname{simplify}(\operatorname{Determinant}(\operatorname{Matrix}([[\mathrm{A}[\mathrm{n}+3], \mathrm{A}[\mathrm{n}+1], \mathrm{A}[\mathrm{n}+2]], \quad[\mathrm{B}[\mathrm{n}+3], \mathrm{B}[\mathrm{n}+1], \mathrm{B}[\mathrm{n}+2]], \quad[\mathrm{C}[\mathrm{n}+3], \mathrm{C}[\mathrm{n}+1]$, $\mathrm{C}[\mathrm{n}+2]]])))$ :

$\mathrm{Z}[\mathrm{n}+2]:=\operatorname{simplify}(\operatorname{Determinant}(\operatorname{Matrix}([[\mathrm{A}[\mathrm{n}+2], \mathrm{A}[\mathrm{n}+1], \mathrm{A}[\mathrm{n}+2]],[\mathrm{B}[\mathrm{n}+2], \mathrm{B}[\mathrm{n}], \mathrm{B}[\mathrm{n}+2]],[\mathrm{C}[\mathrm{n}+2], \mathrm{C}[\mathrm{n}]$, $\mathrm{C}[\mathrm{n}+2]]])))$ :

$\mathrm{Z}[\mathrm{n}+3]:=\operatorname{simplify}(\operatorname{Determinant}(\operatorname{Matrix}([[\mathrm{A}[\mathrm{n}+2], \mathrm{A}[\mathrm{n}+1], \mathrm{A}[\mathrm{n}+3]],[\mathrm{B}[\mathrm{n}+2], \mathrm{B}[\mathrm{n}+1], \mathrm{B}[\mathrm{n}+3]],[\mathrm{C}[\mathrm{n}+2], \mathrm{C}[\mathrm{n}+1]$,

$\mathrm{i}:=$ 'i': $\mathrm{C}[\mathrm{n}+3]]])))$

for $\mathrm{i}$ from 1 to $\mathrm{n}$ do

$$
\begin{aligned}
& \mathrm{S}[\mathrm{i}, \mathrm{n}]:=-\mathrm{Z}[\mathrm{i}] / \mathrm{Z}[\mathrm{n}+3]: \\
& \mathrm{S}[\mathrm{i}, \mathrm{n}-1]:=-\mathrm{Y}[\mathrm{i}] / \mathrm{Y}[\mathrm{n}+2]: \\
& \mathrm{S}[\mathrm{i}, \mathrm{n}-2]:=-\mathrm{X}[\mathrm{i}] / \mathrm{X}[\mathrm{n}+1]:
\end{aligned}
$$

end do:

$\mathrm{i}:=$ 'i':

for i to $\mathrm{n}$ do

$\mathrm{S}[\mathrm{i}, \mathrm{n}-3]:=-\operatorname{simplify}\left(\left(\mathrm{d}[\mathrm{n}]{ }^{*} \mathrm{~S}[\mathrm{i}, \mathrm{n}]+\mathrm{e}[\mathrm{n}-1]^{*} \mathrm{~S}[\mathrm{i}, \mathrm{n}-1]+\mathrm{f}[\mathrm{n}-2]^{*} \mathrm{~S}[\mathrm{i}, \mathrm{n}-2]\right) / \mathrm{g}[\mathrm{n}-3]\right) ;$

if $\mathrm{i}=\mathrm{n}$ then

$$
\mathrm{S}[\mathrm{i}, \mathrm{n}-3]:=\operatorname{simplify}(1 / \mathrm{g}[\mathrm{n}-3]+\mathrm{S}[\mathrm{i}, \mathrm{n}-3]) \text { : }
$$

end if

end do:

if $\mathrm{n}=5$ then

$$
\mathrm{i}:=\text { 'i': }
$$

for i to $\mathrm{n}$ do

$\mathrm{S}[\mathrm{i}, \mathrm{n}-4]:=-\operatorname{simplify}\left(\left(\mathrm{c}[\mathrm{n}] * \mathrm{~S}[\mathrm{i}, \mathrm{n}]+\mathrm{d}[\mathrm{n}-1]^{*} \mathrm{~S}[\mathrm{i}, \mathrm{n}-1]+\mathrm{e}[\mathrm{n}-2]^{*} \mathrm{~S}[\mathrm{i}, \mathrm{n}-2]+\right.\right.$ $\left.\left.\mathrm{f}[\mathrm{n}-3]^{*} \mathrm{~S}[\mathrm{i}, \mathrm{n}-3]\right) / \mathrm{g}[\mathrm{n}-4]\right)$;

$$
\begin{aligned}
& \text { if } \mathrm{i}=\mathrm{n}-1 \text { then } \\
& \qquad \mathrm{S}[\mathrm{i}, \mathrm{n}-4]:=\operatorname{simplify}(1 / \mathrm{g}[\mathrm{n}-4]+\mathrm{S}[\mathrm{i}, \mathrm{n}-4])
\end{aligned}
$$

end if

end do

elif $n=6$ then

$$
\mathrm{i}:=\text { 'i': }
$$

for i to $\mathrm{n}$ do

$\mathrm{S}[\mathrm{i}, \mathrm{n}-5]:=-\operatorname{simplify}\left(\left(\mathrm{b}[\mathrm{n}]^{*} \mathrm{~S}[\mathrm{i}, \mathrm{n}]+\mathrm{c}[\mathrm{n}-1]^{*} \mathrm{~S}[\mathrm{i}, \mathrm{n}-1]+\mathrm{d}[\mathrm{n}-2]^{*} \mathrm{~S}[\mathrm{i}, \mathrm{n}-2]+\mathrm{e}[\mathrm{n}-3]^{*} \mathrm{~S}[\mathrm{i}, \mathrm{n}-3]+\right.\right.$ $\mathrm{f}[\mathrm{n}-3] \mathrm{f}[\mathrm{n}-4] * \mathrm{~S}[\mathrm{i}, \mathrm{n}-4]) / \mathrm{g}[\mathrm{n}-5])$; 


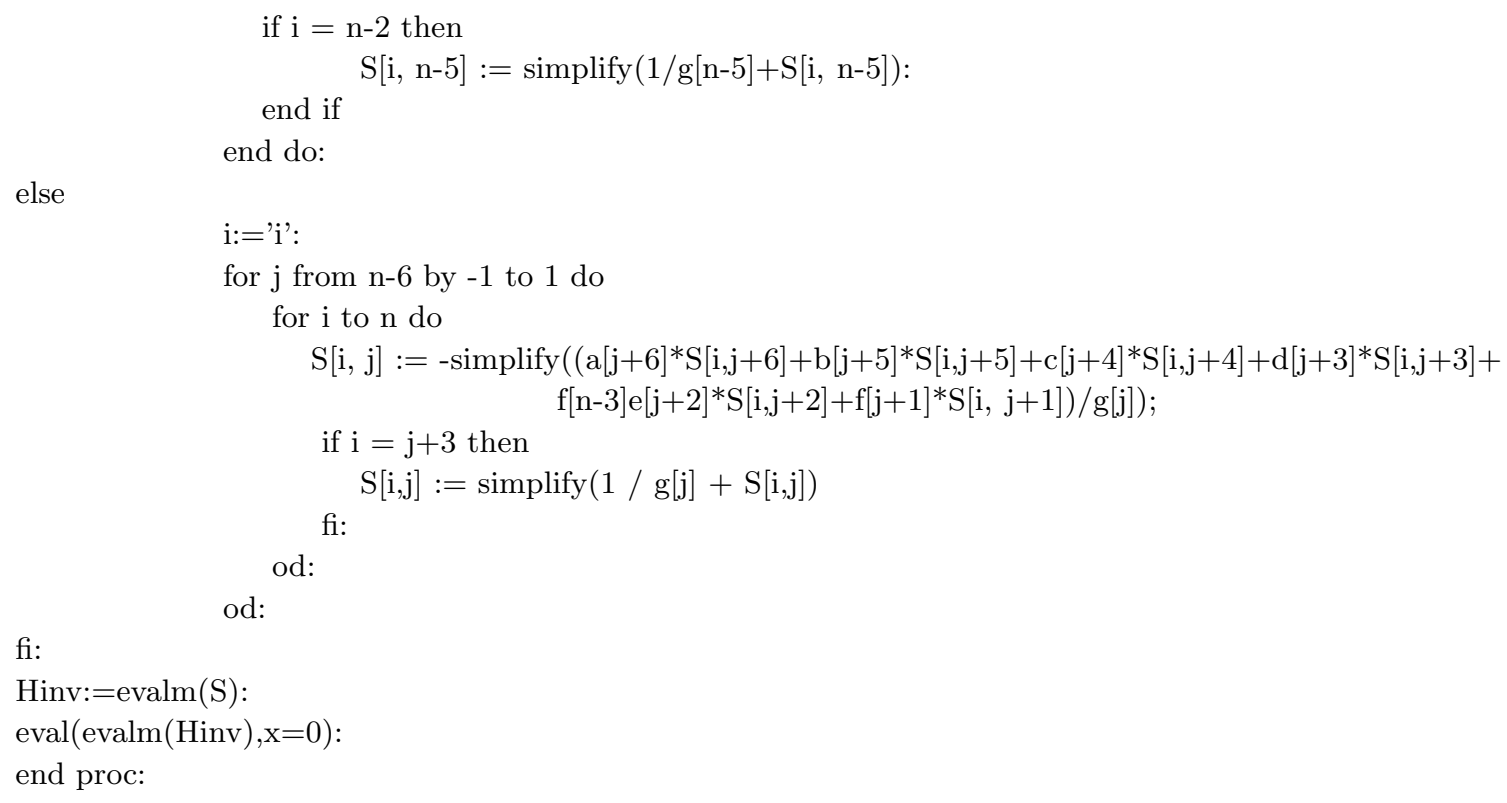

\section{References}

[1] A. Böttcher, S. Grudsky, Spectral Properties of Banded Toeplitz Matrices, SIAM, Philadelphia, 2005.

[2] G.H. Golub, C.F. Van Loan, Matrix Computations, third ed., The Johns Hopkins University Press, Baltimore and London, 1996.

[3] R.L. Burden, J.D. Faires, Numerical Analysis, seventh ed., Books \& Cole Publishing, Pacific Grove, CA, 2001.

[4] T. Yamamoto, Y. Ikebe, Inversion of band matrices, Linear Algebra Appl. 24 (1979) 105-111.

[5] W.F. Trench, An algorithm for the inversion of finite Toeplitz matrices, J. SIAM 12 (1964) 515-522.

[6] M. Elouafi, A note for an explicit formula for the determinant of pentadiagonal and heptadiagonal symmetric Toeplitz matrices, Appl. Math. Comput. 219 (2013) 4789-4791.

[7] M. Solary, Finding eigenvalues for heptadiagonal symmetric Toeplitz matrices, J. Math. Anal. Appl. 402 (2013) 719-730.

[8] D. Ting, M. Gu, X. Chi, J. Cao, Numerical Acceleration of Three-Dimensional Quantum Transport Method Using a Seven-Diagonal Pre-Conditioner, J. Comput. Electron. 1(2002) 93-97.

[9] S. Gu, J. Peng, R. Cui, A Polynomial Time Solvable Algorithm to Binary Quadratic Programming Problems with Q Being a Seven-Diagonal Matrix and Its Neural Network Implementation, Advances in Neural Networks ISNN 2014, Lecture Notes in Computer Science (2014) 338-346.

[10] A. A. Karawia, Inversion of General Cyclic Heptadiagonal Matrices, Math. Probl. Eng. Volume 2013, Article ID 321032, 9 pages.

[11] M. El-Mikkawy, A. A. Karawia, Inversion of general tridiagonal matrices, Appl. Math. Lett. 19 (2006) 712-720.

[12] M. El-Mikkawy, On the inverse of a general tridiagonal matrix, Appl. Math. Comput. 150 (2004) 669-679.

[13] A. Hadj, M. Elouafi, A fast numerical algorithm for the inverse of a tridiagonal and pentadiagonal matrix, Appl. Math. Comput. 202 (2008) 441-445. 